\title{
REDES ECUMÉNICAS A MEDIADOS DEL SIGLO XX. UN ANÁLISIS DE REDES DE COAUTORÍAS EN LOS ESCRITOS DE MAURICIO LÓPEZ (1945-1972) ${ }^{1}$
}

\author{
Alejandro Paredes $\left(^{*}\right)$
}

\section{RESUMEN}

Mauricio López, filósofo y doctor en teología, fue secretario para América Latina de la Federación Universal de Movimientos Estudiantiles Cristianos (FUMEC), secretario adjunto del Departamento de Iglesia y Sociedad del Consejo Mundial de Iglesias (CMI) y rector de la Universidad Nacional de San Luis (Argentina). En 1977 fue secuestrado; es un desaparecido de la última dictadura militar.

Este artículo busca reconstruir una red de intelectuales ecuménicos a partir del análisis de la red de coautoría de Mauricio López. Para ello se analizarán 29 publicaciones, escritas entre 1945 y 1972, en la que intervienen 92 coautores utilizando la metodología del Análisis de Redes sociales (ARS) y los softwares UCINET 6 y Netdraw.

Palabras clave:

Redes de coautoría - ecumenismo - Mauricio López.

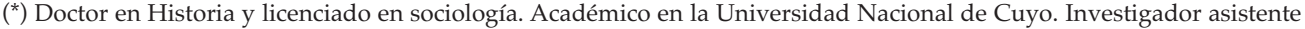
de CONICET, en el Centro Científico Tecnológico de la ciudad de Mendoza, Argentina.

Artículo recibido el 19 de marzo de 2010. Aceptado por el Comité Editorial el 23 de julio de 2010.

Correo electrónico: aparedes@mendoza-conicet.gob.ar

1 Trabajo realizado en el marco del proyecto "Estudio histórico de las redes intelectuales-literarias en América Latina. Secuencias, contactos, configuraciones, religaciones transnacionales y el impacto en la producción letrada" dirigido por el Dr. Claudio Maiz. Proyecto de Investigación Plurianual No 112-200801-01336 (CONICET, Argentina).
} 


\begin{abstract}
Mauricio Lopez, philosopher and doctor of theology, was Secretary for Latin America of the Universal Federation of Student Christian Movements (WSCF), secretary of the Department of Church and Society of the World Council of Churches (WWC) and Chancellor of the Universidad Nacional de San Luis (Argentina). In 1977 he was kidnapped is a desaparecido (missing) of the last military dictatorship.

This article seeks to reconstruct an ecumenical network of intellectuals from the analysis of the Mauricio Lopez's coauthorship network. We will analyze 29 publications, written between 1945 and 1972, which involved 92 co-authors using the methodology of social network analysis (SNA) and the software UCINET 6 and Netdraw.
\end{abstract}

\title{
Keywords:
}

Coauthorship networks - ecumenism - Mauricio Lopez.

\section{INTRODUCCIÓN}

Este artículo se propone analizar una red entre intelectuales ecuménicos de mediados del siglo XX a partir de las colaboraciones en publicaciones de un militante ecuménico llamado Mauricio López. Las redes de coautoría han demostrado ser eficaces para analizar las vinculaciones entre intelectuales contemporáneos (entre otros pueden citarse a: Alcaide, Alonso, González de Dios, Sempere, Valderrama, y Benavent 2008; Chiroque y Padilla, 2009; Alcaide, Agulló, Valderrama y Benavent, 2009; Molina, Muñoz y Domenech, 2002) y también descubrir Colegios invisibles entre intelectuales. Este concepto remite a comunidades informales de personas que estudian un mismo tema, se relacionan entre sí e intercambian información (Iñiguez, Muñoz, Peñaranda y Martínez, 2006).

En este caso se reconstruyó una red de coautoría a partir de la producción de un militante ecuménico que fue muy importante entre las décadas de 1940 y 1970 por su influencia sobre intelectuales, que luego darán origen a la teología y filosofía de la liberación. Su importancia sin embargo, no está acompañada por estudios de su obra y su legado. Principalmente porque su actividad intelectual estuvo asistida por una intensa militancia ecuménica ${ }^{2}$, que en cierto modo ensombreció a la primera. Por esta razón, la primera tarea fue rastrear sus obras. Las fuentes primarias de este artículo son veintinueve publicaciones de Mauricio López en español, inglés y alemán, encontradas en el Consejo Mundial de Iglesias (Ginebra- Suiza), en el Instituto Iberoamericano de Berlín (Alemania) y en el ISEDET (Buenos Aires-Argentina). En estas publicaciones participaron 92 coautores.

\footnotetext{
${ }^{2}$ Que implicaron viajes a Cuba durante el proceso revolucionario, a países de Europa del Este y ayuda a exiliados latinoamericanos.
} 
El Ecumenismo se refiere a las actividades que buscan la unidad entre las religiones cristianas. Dadas las características ideológicas de Mauricio López su red de coautorías nos vinculará con una red de intelectuales cristianos de izquierda de esos años. La metodología utilizada es la del Análisis de Redes Sociales (ARS). Tomando a todas las publicaciones de Mauricio López encontradas se vincularon a los coautores de un mismo texto, en una matriz binaria del software UCINET 6 y se reconstruyó la red en un grafo por medio de Netdraw. Para los efectos de este trabajo y a fin de la reconstrucción de una red de intelectuales de una época específica, se entiende por coautoría a la publicación conjunta de un artículo y a la participación de varios autores en un libro (aunque esto implique que cada autor haya escrito un capítulo individual).

\section{EL CASO DE MAURICIO LÓPEZ}

Partiendo de la obra de un filósofo y teólogo latinoamericano, Mauricio López, el artículo busca mostrar la red político-religiosa entre las décadas de 1940 y 1970. Mauricio López nació en Bahía Blanca (Argentina) fue licenciado en filosofía en la Universidad Nacional de Cuyo (Mendoza, Argentina) y doctor en teología en la Facultad de Teología Protestante de París (Francia). Entre otras tareas fue secretario para América Latina de la Federación Universal de Movimientos Estudiantiles Cristianos (FUMEC) entre 1955 y 1963, secretario adjunto del Departamento de Iglesia y Sociedad del Consejo Mundial de Iglesias (1963-1968), Miembro fundador de Iglesia y Sociedad en América Latina (ISAL) y rector de la Universidad Nacional de San Luis (Argentina) desde 1973, pero quedó cesante luego del golpe militar de Argentina de 1976. Tuvo un heroico compromiso en la ayuda a chilenos exiliados en Argentina después del golpe de 1973 y con perseguidos políticos argentinos después del golpe militar de 1976. En la noche del año nuevo de 1977 fue secuestrado y es un desaparecido más de la última dictadura militar argentina. Posiblemente estuvo cautivo en el campo clandestino de detención de "Las Lajas", en la provincia de Mendoza, dónde murió víctima de la tortura (Paredes, 2008, pp. 5-19).

\section{SU PRODUCCIÓN ESCRITA}

Entre 1945 y 1972, López publicó 29 trabajos que son analizados en el siguiente cuadro: 


\begin{tabular}{|c|c|c|c|}
\hline & Cuadro $\mathbf{N}^{\circ} 1$ & & \\
\hline & Análisis de las publicaciones de Ma & uricio López. & \\
\hline Año & Artículos & Capítulos de libro & $\mathrm{N}^{0}$ \\
\hline $1945 / 47$ & 6 Reseñas de libros de filosofía & - & 6 \\
\hline 1952 & El dato noético inicial de la filosofía & - & 1 \\
\hline 1954 & Academic life, political community a state intervention & - & 1 \\
\hline 1956 & Latin America notes & - & 1 \\
\hline 1957 & American travel diary (I); Spanish books; Of Hope and Promise & - & 3 \\
\hline 1958 & $\begin{array}{c}\text { American travel diary (II); General view of the Latin American } \\
\text { situation; The Federation in Latin America }\end{array}$ & - & 3 \\
\hline 1959 & $\begin{array}{l}\text { Iberian and Caribbean travel diary; Latin American student and } \\
\text { their participation in political life; Theological discussions in the } \\
\text { Caribbean area }\end{array}$ & - & 3 \\
\hline 1962 & $\begin{array}{l}\text { The letter that arrived late; The university and Christian witness } \\
\text { in Latin America setting }\end{array}$ & - & 2 \\
\hline 1963 & $\begin{array}{c}\text { A New Protestant Generation Meets in the Aztec Capital; De las } \\
\text { revoluciones a la revolución }\end{array}$ & - & 2 \\
\hline 1964 & Annotated bibliography on Latin America & - & 1 \\
\hline 1966 & - & $\begin{array}{l}\text { The political dynamics of Latin } \\
\text { American society today }\end{array}$ & 1 \\
\hline 1967 & - & The church amid revolution & 1 \\
\hline 1969 & - & Kulturelle wertbegriffe & 1 \\
\hline 1971 & - & $\begin{array}{l}\text { La liberación de América Latina y el } \\
\text { cristianismo evangélico; El contexto } \\
\text { secular de la misión: La América } \\
\text { Latina de la década del } 60\end{array}$ & 5 \\
\hline 1972 & - & $\begin{array}{l}\text { Independence, Development and } \\
\text { Liberation in Latin America* }\end{array}$ & 1 \\
\hline Total & 23 & 6 & 29 \\
\hline $\begin{array}{l}\text { * Publi } \\
\text { Fuentes: }\end{array}$ & $\begin{array}{l}\text { do en alemán e inglés. } \\
\text { ablicaciones de Mauricio López rastreadas en el Consejo } 1 \\
\text { eroamericano de Berlín (Alemania) e ISEDET (Buenos Air }\end{array}$ & $\begin{array}{l}\text { ndial de Iglesias (Ginebra- Suiza } \\
\text {-Argentina). }\end{array}$ & \\
\hline
\end{tabular}


Como puede apreciarse, el $65 \%$ de las publicaciones fueron editadas en inglés y eran artículos en revistas. Los temas predominantes fueron la situación social en Latinoamérica, FUMEC y los resultados de sus viajes (el 70\% de las publicaciones se centraron en estos tópicos). Entre 1955 y 1963, muchos de sus artículos fueron publicados en las revistas Federation News, (Laussane, Suiza) y The Student World (Ginebra, Suiza). En la primera, publicó cinco artículos sobre FUMEC en Latinoamérica y también sobre la situación universitaria en general. The Student World publicó ocho artículos de López, cuatro de ellos se refieren a sus viajes como secretario para América Latina de FUMEC; un artículo es un estudio de libros y los tres restantes se detienen en la situación social latinoamericana. El 31\% de las publicaciones fueron en español, dos eran artículos, uno en la revista Cristianismo y Sociedad de Montevideo y otro en la Revista del Instituto de Filosofía, de la Universidad Nacional de Cuyo llamada Philosophia. En esta última también publicó seis reseñas de libros y tradujo al español un artículo de A.H. Johnson sobre la filosofía de la historia en Whitehead (esta traducción no aparece en el cuadro porque la autoría es de Johnson y no de López). Tampoco aparece en el cuadro una conferencia dada en inglés llamada "The gospel and the intellectual world" que aborda la problemática entre fe y razón y se dio en el marco I Conferencia Latinoamericana del LMC (Life and Mission of the Church) y de la Consulta Política Interamericana sobre la situación en Norte y Sudamérica en 1963. No fue incluida porque la conferencia no fue publicada como tal, sin embargo, Mauricio López y varios autores que participaron en el LMC, luego publicaron el libro ¿Misioneros de Estados Unidos para qué? (1971) en el que aparecen las reflexiones abordadas en esa reunión del LMC. Finalmente, sólo dos capítulos de libro son en alemán "Kulturelle wertbegriffe" que forma parte del libro Explosives lateinamerika. Der Protestantismus inmitten der Sozialen Revolution y la versión en alemán de "Independence, Development and Liberation in Latin America" en el libro Christen im weltweiten Friedenskampf: Studienband der Christlichen Friedenskonferenz. Sobre ellos nos detendremos al analizar los libros en coautoría.

\section{SUS VIAJES}

Gran parte de su vinculación con intelectuales ecuménicos (cristalizada en trabajos en coautorías) fue tejida en sus numerosos viajes. Entre 1955 y 1963, Mauricio López fue designado secretario para América Latina de la Federación Universal de Movimientos Estudiantiles Cristianos (FUMEC). En ese periodo visitó 34 países, reuniéndose con organizaciones cristianas y llevando a cabo distintas actividades, principalmente retiros espirituales, seminarios para líderes comunitarios y conferencias en universidades. Hacia 1963 FUMEC en América Latina experimentó el mayor crecimiento desde su creación (Potter y Wieser, 1997). Sus viajes eran unas giras de varios meses que se detallan a continuación: 


\begin{tabular}{|c|c|c|c|c|c|}
\hline \multicolumn{6}{|c|}{$\begin{array}{c}\text { Cuadro } \mathrm{N}^{\circ} 2 \\
\text { Viajes de Mauricio López como secretario para América Latina de FUMEC } \\
(1955-1963) .\end{array}$} \\
\hline \multirow[t]{2}{*}{ Viaje } & \multirow[t]{2}{*}{ Año } & \multicolumn{4}{|c|}{$\begin{array}{rr} & \text { País }\end{array}$} \\
\hline & & América Latina & Asia & Europa & $\begin{array}{l}\text { Norte } \\
\text { América }\end{array}$ \\
\hline & 1956 & Bolivia & - & - & - \\
\hline & 1957 & $\begin{array}{l}\text { México, Cuba, Jamaica, República Domini- } \\
\text { cana, Puerto Rico y Brasil. }\end{array}$ & - & - & - \\
\hline & 1957 & $\begin{array}{l}\text { Chile, Perú, Ecuador, Paraguay, Bolivia, } \\
\text { Uruguay y provincias Argentinas. }\end{array}$ & - & - & - \\
\hline & 1958 & $\begin{array}{l}\text { Chile, Perú, Ecuador, Bolivia, Paraguay, } \\
\text { Uruguay, Argentina y Guatemala }\end{array}$ & - & $\begin{array}{l}\text { Suiza, Bélgica y } \\
\text { Dinamarca. }\end{array}$ & $\begin{array}{l}\text { EE.UU. } \\
\text { Canadá. }\end{array}$ \\
\hline & 1959 & $\begin{array}{l}\text { Argentina, Venezuela, Colombia, Perú, } \\
\text { Uruguay, Brasil, Cuba, Jamaica, Puerto } \\
\text { Rico, Haití, República Dominicana, Suri- } \\
\text { nam y Costa Rica, }\end{array}$ & - & $\begin{array}{l}\text { Portugal, España, } \\
\text { Alemania y Suiza. }\end{array}$ & - \\
\hline & 1960 & Costa Rica & - & $\begin{array}{l}\text { Portugal, España, Suiza, } \\
\text { Checoslovaquia }\end{array}$ & EE.UU. \\
\hline & 1960 & $\begin{array}{l}\text { México, Guatemala, El Salvador, Hondu- } \\
\text { ras, Nicaragua, Costa Rica, Panamá, Para- } \\
\text { guay y Argentina. }\end{array}$ & - & - & EE.UU. \\
\hline & 1961 & $\begin{array}{l}\text { Cuba, República Dominicana, Puerto Rico, } \\
\text { Brasil y Argentina. }\end{array}$ & & $\begin{array}{l}\text { Portugal, España, } \\
\text { Francia, y Bélgica }\end{array}$ & EE.UU. \\
\hline & 1962 & $\begin{array}{l}\text { México, Cuba, Jamaica, República Domi- } \\
\text { nicana, Puerto Rico, Venezuela, Uruguay, } \\
\text { Paraguay, Bolivia y Argentina }\end{array}$ & India & Bélgica & EE.UU. \\
\hline & 1962 & $\begin{array}{l}\text { Chile, Perú, Colombia, Costa Rica, México } \\
\text { y Brasil }\end{array}$ & - & - & - \\
\hline & 1963 & Uruguay & - & Suiza & - \\
\hline & 1968 & - & - & Francia & - \\
\hline & 1969 & Argentina & - & - & - \\
\hline $\begin{array}{l}\text { Total } \\
1956-1\end{array}$ & $\begin{array}{l}\text { viajes } \\
69\end{array}$ & $\begin{array}{c}23 \\
68 \%\end{array}$ & $\begin{array}{c}1 \\
3 \%\end{array}$ & $\begin{array}{c}8 \\
23 \%\end{array}$ & $\begin{array}{c}2 \\
6 \%\end{array}$ \\
\hline
\end{tabular}

Entre otras actividades, en estos viajes dio cursos a dirigentes sociales en Perú y Argentina (1958), preparó conferencias para el Primer Retiro Espiritual de Estudiantes Universitarios de América Central (Guatemala, 1958). Dio cursos de estudio bíblico para dirigentes sudamericanos (Sao Paulo, 1959). Preparó el Congreso para Estudiantes de Teología del Caribe (Jamaica, 1959). Dio un importante apoyo a las iglesias cubanas luego de la revolución castrista, ingresando a la isla vía Europa del Este después del bloqueo estadounidense. Participó en el Curso para dirigentes de ULAJE: Unión Latinoamericana de Juventud Evangélica (Costa Rica, 1960). Dio una conferencia en la Universidad de Pittsburg (1962). Organizó la I Conferencia Latinoamericana del LMC (Life and Mission of the Church) y la Consulta Política Interamericana sobre la situación en Norte y Sudamérica (ambas en México, 1962). 
Con respecto a las organizaciones europeas, además de estar por largos periodos en Ginebra vinculando las actividades de FUMEC y el Consejo Mundial de Iglesias (CMI), organizó actividades con movimientos ecuménicos de Bélgica, Dinamarca, España, Francia, Portugal y las, entonces Checoslovaquia, URSS y Yugoslavia. Dictó un curso para dirigentes estudiantiles en Caravelos (Portugal, 1961) y representó a FUMEC en reuniones con estudiantes latinoamericanos católicos y protestantes que estudiaban en Europa en la Universidad Católica de Lovaina (Bélgica, 1961-1962).

Los últimos tres viajes no fueron registrados detalladamente como los anteriores por el archivo de FUMEC de Ginebra. Sabemos que en 1962 fue al encuentro de la India del Consejo Mundial de Iglesias (CMI) representando a FUMEC. Allí fue elegido secretario adjunto de Iglesia y Sociedad. Al año siguiente luego de un reunión en Uruguay se mudó a Ginebra para trabajar en el CMI, donde permaneció hasta 1968 (es el viaje número 11 en el cuadro anterior). En 1968 se mudó a Francia para realizar sus estudios doctorales en la Facultad Libre de Teología Protestante de París. Al año siguiente regresó a Argentina donde se dedicó a la docencia universitaria hasta ser designado rector de la Universidad Nacional de San Luis. De todos modos siguió vinculado a su militancia ecuménica a través de su participación en el programa de Conferencia para la Paz Cristiana del CMI y de ISAL (Iglesia y Sociedad para América Latina).

\section{LA RED DE COAUTORÍAS DE MAURICIO LÓPEZ}

Las publicaciones de Mauricio López son fruto de su militancia ecuménica. Sus participaciones en obras colectivas son reflexiones a partir de congresos, retiros y conferencias junto a otros militantes ecuménicos de otros países (esto explica la baja presencia de argentinos en su red de coautoría). Por esta razón, muchos de sus escritos son una sociología de la religión que se detiene en aspectos de los movimientos religiosos y políticos que el autor vivía en ese momento en latinoamericana. El siguiente cuadro detalla las publicaciones en coautorías encontradas en las obras de Mauricio López: 


\section{Cuadro $\mathbf{N}^{\circ} 3$}

\section{Cuadro de coautores con Mauricio López por publicación.}

\begin{tabular}{|c|c|c|c|}
\hline Publicación ${ }^{*}$ & año & Autores & $\mathrm{N}^{0}$ \\
\hline $\begin{array}{l}\text { A New Protestant Generation } \\
\text { Meets in the Aztec Capital }\end{array}$ & 1963 & J Nieto y Mauricio López & 2 \\
\hline $\begin{array}{l}\text { The political dynamics of Latin } \\
\text { American society today }\end{array}$ & 1966 & $\begin{array}{l}\text { Z.K.Matthews, M.M. Thomas, Helmut Gollwitzer, Max Kohn- } \\
\text { stamm, Kenneth W. Thompson, John H. Hallowell, J.B. Soucek, } \\
\text { Chandran Devanesen, André Philip, Peter D. Latuihamallo, Rich- } \\
\text { ard Andriamanjato, A.F. Carrillo de Albornoz, Sean MacBride, T.B. } \\
\text { Simatupang, Henri Burgelin, John Karefa-Smart, M. Abel y Mauri- } \\
\text { cio López. }\end{array}$ & 18 \\
\hline The church amid revolution & 1967 & $\begin{array}{l}\text { A. Delobelle, André Dumas, César Espíritu, François Abbé Houtart, } \\
\text { J.M. Lochman, Roger L.Shinn, Massao Takenaka, Charles C.West, } \\
\text { Mauricio López M.M.Thomas, John Karefa-Smart, Ricardo Shaull } \\
\text { y Emilio Castro. }\end{array}$ & 13 \\
\hline $\begin{array}{l}\text { Explosives lateinamerika. Der } \\
\text { Protestantismus inmitten der } \\
\text { Sozialen Revolution }\end{array}$ & 1969 & $\begin{array}{l}\text { Mauricio López Hiber Conteris, Julio de Santa Ana, Rubem A.Alves, } \\
\text { Emilio Castro, Eugene Nida, Enrique Dussel, Rudolf Obermüller, } \\
\text { Philip Potter, Thomas Liggett, Walter Hollenweger, Valdo Galland, } \\
\text { Waldo Cesar, José Miguez Bonino, Héctor Borrat, Adolfo Ham, Os- } \\
\text { car Bolioli, Nestor Garcia Canclini, Luis Odell, Jorge Lara-Braud y } \\
\text { Samuel Araya. }\end{array}$ & 21 \\
\hline De la iglesia y la sociedad & 1971 & $\begin{array}{l}\text { Rubem A Alves, Ricardo Shaull, Leopoldo Niilus, Mauricio López, } \\
\text { Julio Barreiro, Pierre Furter, Julio de Santa Ana, Gonzalo Castillo, } \\
\text { Waldo Villalpando, Christian Lalive, Sergio Arce Martínez, Hiber } \\
\text { Conteris y Theo Tschuy. }\end{array}$ & 13 \\
\hline $\begin{array}{l}\text { Misioneros estadounidenses } \\
\text { ¿para qué? }\end{array}$ & 1971 & $\begin{array}{l}\text { Emilio Castro, Joao da Silva, Alfredo Ramírez, Daily Resende Fran- } \\
\text { ça, Eugene L.Stockwell, Edmundo Desueza y M. López. }\end{array}$ & 7 \\
\hline $\begin{array}{l}\text { Christians today in the } \\
\text { struggle for peace and social } \\
\text { justice } \\
\text { (también publicado en alemán } \\
\text { en } 1972 \text { con el título: Christen } \\
\text { im weltweiten Friedenskampf: } \\
\text { Studienband der Christlichen } \\
\text { Friedenskonferenz) }\end{array}$ & 1972 & $\begin{array}{l}\text { Károly Tóth, Gerhad Bassarak, Pavel S. Sokolovsky, Tibor Bartha, } \\
\text { Károly Pröhle, Manfred Haustein, Janusz Makowski, Otto Hartmut } \\
\text { Fuchs, A.J. Koejemans, Karol Gábris, Rosemarie Müller-Streisand, } \\
\text { Metropolitan Nikodim, Paul Verghese, Eduardo Coloma, Keiishi } \\
\text { Kobayashi, Gonzalo Arroyo, Heinrich Werner, Elemér Kocsis, Wal- } \\
\text { ter Bredendiek, Pham Quang Phuoc, Alexi Stoyan, Maurice Tadros, } \\
\text { renate Riemeck, Ralph David Abernathy, Ambrose Reeves, Todor } \\
\text { Sabev, Slavtcho Valtchanov, Günther Wirth, Simon-Peter Gerlach, } \\
\text { Carl Soule, Mauricio López y Richard Andriamanjato. }\end{array}$ & 32 \\
\hline \multicolumn{3}{|l|}{ Total } & 106 \\
\hline
\end{tabular}

* aparece el título del libro escrito en coautoría o artículo en coautoría.

Fuentes: Publicaciones de Mauricio López rastreadas en el CMI (Ginebra- Suiza), Instituto Iberoamericano de Berlín (Alemania) e ISEDET (Buenos Aires -Argentina).

Si bien en el cuadro aparecen 106 autores, en realidad son 92 ya que varios de ellos participan en más de una publicación. El siguiente cuadro se detiene en el origen de los autores: 


\section{Cuadro $\mathrm{N}^{\circ} 4$}

\section{Autores por lugar con los que ha escrito Mauricio López.}

\begin{tabular}{|c|c|c|c|}
\hline Lugar & Autores & $\mathrm{N}^{0}$ & $\%$ \\
\hline África & $\begin{array}{l}\text { Z.K. Matthews, Richard Andriamanjato, John Karefa-Smart, Eduardo Coloma y Maurice } \\
\text { Tadros. }\end{array}$ & 5 & 5,4 \\
\hline $\begin{array}{l}\text { América } \\
\text { Latina }\end{array}$ & $\begin{array}{l}\text { Enrique Dussel, Philip Potter, Thomas Liggett, Valdo Galland, Waldo Cesar, José Miguez } \\
\text { Bonino, Héctor Borrat, Adolfo Ham, Oscar Bolioli, Nestor García Canclini, Luis Odell, } \\
\text { Jorge Lara-Braud, Samuel Araya, Rubem A. Alves, Leopoldo Niilus, Mauricio López, } \\
\text { Julio Barreiro, Julio de Santa Ana, Gonzalo Castillo, Waldo Villalpando, Sergio Arce Mar- } \\
\text { tínez, Hiber Conteris, J. Nieto, Gonzalo Arroyo, Emilio Castro, Joao da Silva, Alfredo } \\
\text { Ramírez, Dailv Resende Franca, Edmundo Desueza y I. Nieto. }\end{array}$ & 29 & 31,5 \\
\hline Asia & $\begin{array}{l}\text { M.M. Thomas, Chandran Devanesen, Peter D. Latuihamallo, T.B. Simatupang, M. Abel, } \\
\text { Paul Verghese, Keiishi Kobayashi, Pham Quang Phuoc, César Espíritu y Massao Take- } \\
\text { naka. }\end{array}$ & 10 & 10,9 \\
\hline Europa & $\begin{array}{l}\text { Rudolf Obermüller, Walter Hollenweger, Pierre Furter, Christian Lalive, Theo Tschuy, } \\
\text { Helmut Gollwitzer, Max Kohnstamm, J.B.Soucek, André Philip, A.F.Carrillo de Albor- } \\
\text { noz, Sean MacBride, Henri Burgelin, Károly Tóth, Pavel S. Bassarak, Gerhad Sokolovsky, } \\
\text { Tibor Bartha, Károly Pröhle, Manfred Haustein, Janusz Makowski, Otto Hartmut Fu- } \\
\text { chs, A.J.Koejemans, Karol Gábris, Rosemarie Müller-Streisand, Metropolitan Nikodim, } \\
\text { Heinrich Werner, Elemér Kocsis, Walter Bredendiek, Alexi Stoyan, Renate Riemeck, Am- } \\
\text { brose Reeves, Todor Sabev, Slavtcho Valtchanov, Günther Wirth, Simon-Peter Gerlach, } \\
\text { A.Delobelle, André Dumas, François Abbé Houtart, J.M.Lochman y Charles C.West. }\end{array}$ & 39 & 42,4 \\
\hline Norteamérica & $\begin{array}{l}\text { William Wonderly, Eugene Nida, Ricardo Shaull, Kenneth W.Thompson, John } \\
\text { H.Hallowell, Ralph David Abernathy, Carl Soule, Eugene L.Stockwell y Roger L.Shinn, }\end{array}$ & 9 & 9,8 \\
\hline \multicolumn{2}{|l|}{ Total } & 92 & 100 \\
\hline
\end{tabular}

La importancia de Mauricio López puede ser inferida a través del origen de los coautores ya que esto es un indicador de su impacto. Un $42,9 \%$ son europeos, pocos más de un $40 \%$ son americanos pero llama la atención que el 16,3\% sean autores africanos y asiáticos, los cuales son muy inaccesibles a los latinoamericanos aún hoy. El siguiente grafo (realizado con Netdraw) ilustra cómo es la relación entre los 92 autores, incluido López. En el mismo, cada nodo corresponde a un autor que publicó con M. López y los lazos entre ellos a las vinculaciones entre autores que publicaron en coautoría un mismo texto. La forma del nodo varía según el origen del autor. 


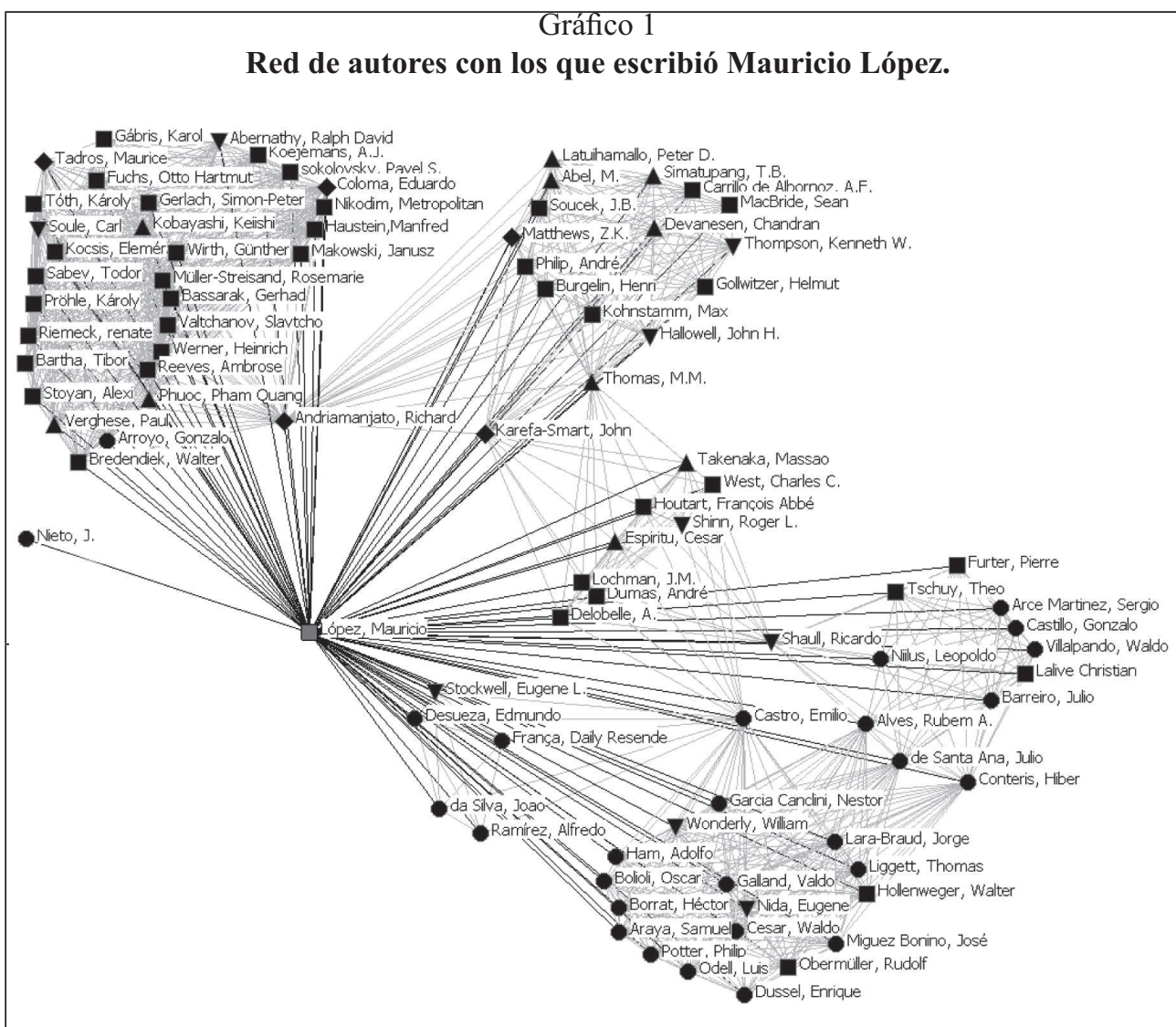

\section{Referencias:}

Forma de los nodos: redondos (latinoamericanos); cuadrados (europeos); rombos (africanos); triángulo hacia arriba (Asia), Triángulo hacia abajo (América anglosajona).

Color de los nodos: gris (Mauricio López); negro (el resto de los autores con los que escribió M. López)

Color de los Lazos: negro (vínculos con M. López); gris (vínculos entre el resto de los autores)

Fuentes: Publicaciones de M. López rastreadas en el Consejo Mundial de Iglesias (GinebraSuiza), Instituto Iberoamericano de Berlín (Alemania) e ISEDET (Buenos Aires -Argentina).

Por supuesto, todos los nodos aparecen vinculados con López, pero si lo retiramos del gráfico podremos apreciar mejor los cliques o subgrupos al interior de la red (ver el gráfico número 2). El cliques es un conjunto de nodos con una fuerte relación interna pero una débil relación con la red a la que pertenecen (Herrero, 2000). En el siguiente gráfico aparecen 6 subgrupos o cliques y un nodo "J. Nieto" que queda aislado del resto de la red porque sólo se vincula con López. El nombre de los subgrupos ha sido puesto según la actividad en conjunto que sirvió de contexto para la creación de la publicación. 
Estos son: Conferencia de Ginebra, Conferencia para la Paz Cristiana, CMI, ISAL- CMI, LMC y FUMEC; en el próximo apartado nos detendremos en ellos. A su vez existen nodos que participan en más de un subgrupo, son los llamados "puntos de corte". Son nodos que al desaparecer dos subgrupos quedan aislados. Puede pensarse en puntos de cortes relativos (porque existe más de un nodo que los vinculan, tal es el caso de Karefa Smart o de Thomas) y puntos de cortes absolutos en los que su ausencia aísla completamente a un subgrupo, por ejemplo Richard Andriamajato y Emilio Castro.

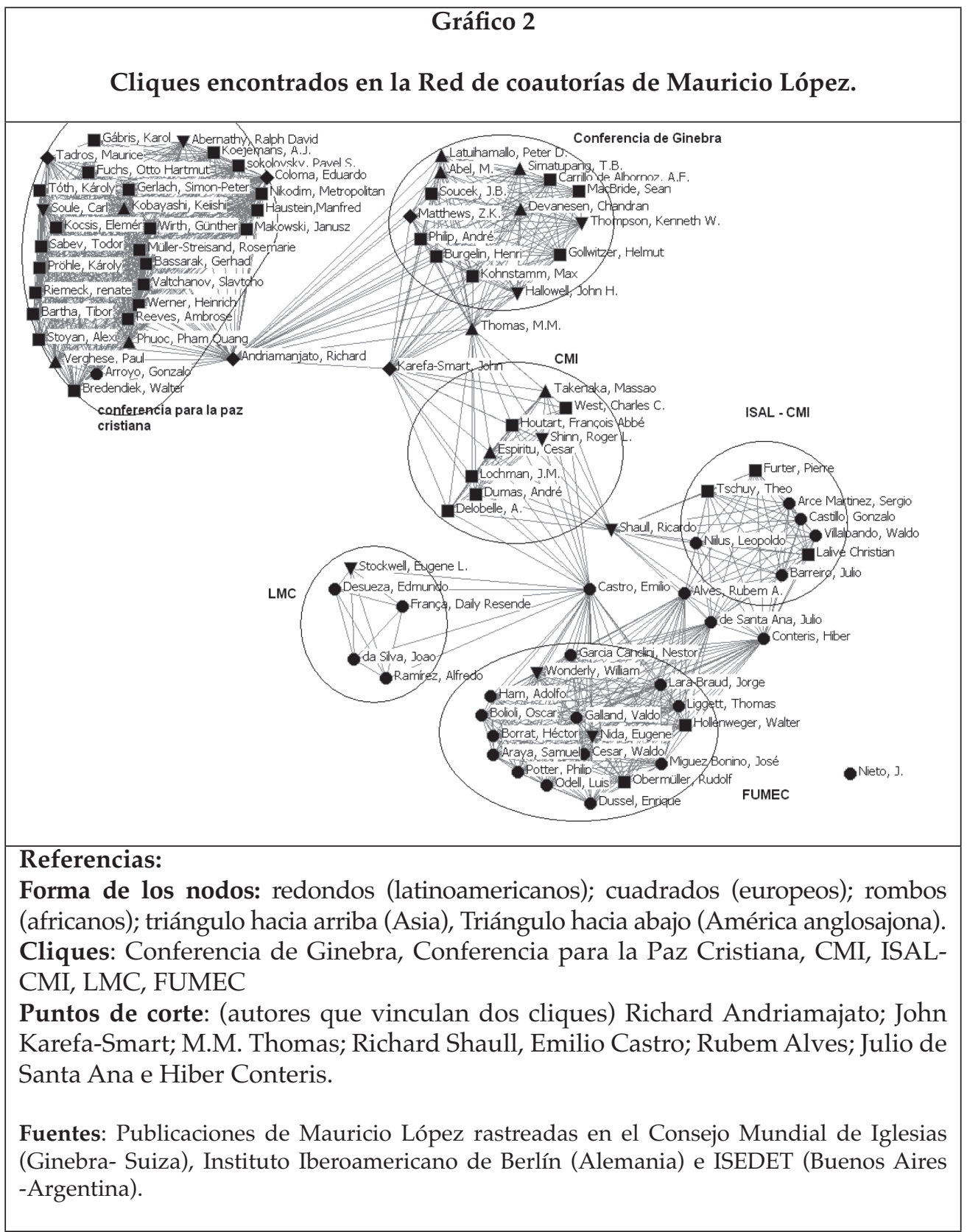


También pueden observarse subgrupos en los que predominan los autores europeos (Conferencia para la Paz Cristiana, Conferencia de Ginebra) y otros en los que predominan los latinoamericanos (ISAL, FUMEC).

\section{CLIQUES AL INTERIOR DE LAS REDES DE COAUTORÍAS}

En este apartado nos detendremos en los subgrupos encontrados: FUMEC, CMI, Conferencia de Ginebra, LMC, ISAL- CMI y Conferencia para la Paz Cristiana.

Como ya se ha señalado López fue secretario adjunto para América Latina de la Federación Universal de Movimientos Estudiantiles Cristianos (FUMEC), entre 1955 y 1963 y la mayoría de sus viajes tuvieron que ver con este rol. Por esta razón no es extraño que éste sea unos de los subgrupos más grandes encontrados en la red. El mismo comprende a estudiantes americanos que participaron, con distintos niveles de compromiso, en agrupaciones cristianas en sus universidades. La excepción son dos europeos en el grupo que también estaban vinculados a los movimientos cristianos de sus países. Algunos de estos jóvenes (como Néstor García Canclini y Enrique Dussel) adquirirán renombre como intelectuales. FUMEC es un organismo que nuclea a los movimientos de estudiantiles cristianos nacionales, que a su vez está compuesto por unidades locales diversas (Potter y Wieser, 1997). FUMEC trabaja vinculado fuertemente con el Consejo Mundial de Iglesias (CMI) y la trayectoria de la militancia de M. López, primero como líder de FUMEC y luego como líder del CMI, es bastante común aún hoy.

El subgrupo llamado CMI hace referencia a los líderes del CMI que vivían en Ginebra. $\mathrm{Su}$ importancia puede inferirse de la centralidad que ocupan en la red. Si el cliques CMI desaparece la red se dividiría en dos: Conferencia de Ginebra y Conferencia para la Paz Cristiana por un lado, y por el otro FUMEC, ISAL-CMI y LMC. Esto no pasaría con la desaparición de ninguno de los otros subgrupos. Su centralidad implica la importancia que tuvo el Consejo Mundial de Iglesias como mediador de recursos y vinculador entre autores.

Como miembro del CMI, López participó en la organización de importantes proyectos y actividades: la Conferencia Mundial de Iglesia y Sociedad y del LMC, y su apoyo en la fundación de ISAL. Estas tareas aparecen reflejadas a través de los subgrupos de autores con los que se relacionó.

Mauricio López, como secretario adjunto del Departamento de Iglesias y Sociedad del Consejo Mundial de Iglesias, organizó junto a Paul Abrecht la Conferencia Mundial de Iglesia y Sociedad, que reunió a los sectores cristianos más progresistas del globo. La misma se llevó a cabo en Ginebra desde el 30 de agosto al 6 de septiembre de 1966. Allí por primera vez se cuestionó el eurocentrismo del pensamiento teológico cristiano y se crearon los Programas de Combate al Racismo, de apoyo a la lucha por la liberación en África y la Participación de las iglesias en el desarrollo (ALC, 2006). En este contexto, López participó en dos libros colectivos: uno introductorio, publicado antes del inicio de la conferencia (The political dynamics of Latin American Society today, 
1966) y otro reflexivo posterior a la misma (The church amid revolution, 1967).

Otra de sus tareas fue la organización de la ya mencionada I Conferencia Latinoamericana del LMC (Life and Mission of the Church) y la Consulta Política Interamericana sobre la situación en Norte y Sudamérica en México. En ella participaron movimientos de América Latina y Anglosajonia que permitieron que años más tarde apareciera el libro con el provocador título "Misioneros estadounidenses ipara qué?".

La fundación de Iglesia y Sociedad en América Latina (ISAL) se inició en 1959, cuando el Departamento de Estudios del CMI estableció en el continente un secretariado, que comenzó a publicar un boletín titulado Iglesia y Sociedad en América Latina. En 1961, en São Paulo se constituyó la Junta Latinoamericana de Iglesia y Sociedad (ISAL) con el apoyo de siete federaciones de iglesias. Entre otros, participaron del movimiento ISAL: Julio de Santa Ana, Richard Shaull, José Miguez Bonino, Hiber Conteris, Emilio Castro, Rubem Alves, Waldo Cesar, Carlos Rodrigues Brandão, Mauricio López y Jaime Wright. Además del asesoramiento de prestigiosos profesionales laicos como Pierre Furter (educador de la Unesco) o Christian Lalive D’Épinay (sociólogo suizo), entre otros. La revista Cristianismo y Sociedad de la Editorial Tierra Nueva fue el órgano de divulgación del movimiento. Este movimiento se orientó hacia posiciones de izquierda cada vez más radicales y muchos de sus miembros optaron por la vía revolucionaria del cambio social (de Santa Ana, 2007). La persecución de sus miembros (que implicó cárcel, exilios y desapariciones) luego de los golpes militares del Cono Sur Americano contribuyó a su decadencia a mediados de los setenta (de Carvalho Dias 2007, pp. 192-195).

El último subgrupo "Conferencia para la Paz Cristiana" es el más numeroso y el más expandido en el globo ya que tiene representantes de África, América, Asia y Europa. Desde 1970 hasta su desaparición, fue también un miembro activo de la Conferencia de Paz Cristiana (Christian Peace Conference). Para ese entonces, se había mudado a la Argentina y ya no era secretario adjunto del Departamento de Iglesias y Sociedad del CMI. Como resultado de ello, pueden leerse dos publicaciones (una en inglés y otra en alemán) que coordinó junto a Gerhard Bassarak: Christians today in the struggle for peace and social justice (Cristianos hoy en la lucha para la paz y la justicia social) y Christen im weltweiten Friedenskampf: Studienband der Christlichen Friedenskonferenz (Cristianos en la lucha mundial de la paz: Estudio del volumen de la Conferencia Cristiana de la Paz).

\section{PUNTOS DE CORTES}

En este apartado nos centraremos en los autores que vinculan a los distintos subgrupos, llamados puntos de corte. Los encontrados en la red de coautoría son los latinoamericanos: Julio de Santa Ana, Rubem Alves, Hiber Conteris y Emilio Castro; los africanos John Karefa-Smart y Richard Andriamanjato; el estadounidense Richard Shaull y el indio Madathilparampil Mammen Thomas. A continuación nos detendremos en cada uno de ellos.

Julio de Santa Ana es uruguayo, estudió teología en Buenos Aires y filosofía en Montevideo. En 1960 obtuvo una beca del Consejo Mundial de Iglesias (CMI) para 
realizar su doctorado en teología en la Universidad de Estrasburgo. Se recibió en 1962. En 1963 comenzó a trabajar como secretario de la Federación de Iglesias Evangélicas del Uruguay, director del Centro de Estudios Cristianos del Río de la Plata, Secretario de ISAL y como editor de la revista Cristianismo y Sociedad (publicación del movimiento ISAL). Luego del rompimiento de la democracia en Uruguay se exilió en Ginebra, donde siguió trabajando en el CMI. Allí fundó la Comisión sobre la Participación de Iglesias en el Desarrollo, en cuyo nombre viajó a Costa Rica y se reunió con el teólogo brasileño Hugo Assmann. Posteriormente, Assmann fundó el Departamento Ecuménico de Investigaciones (DEI) y Santa Ana se vinculó fuertemente a esta institución. Más tarde, Santa Ana fue profesor de religión y ciencias sociales en la Universidad Metodista de São Paulo, donde también se desempeñó como director adjunto del Centro Ecuménico de Evangelización y Educación Popular (CESEP). En 1994 regresó a enseñar al Instituto Ecuménico de Bossey.

Rubem Alves es un expastor presbiteriano brasileño. Magíster en teología, doctor en filosofía del Seminario Teológico de Princeton (EE.UU.) y psicoanalista. Fue uno de los fundadores de la llamada, Teología de la Liberación. Por sus nuevas ideas se le prohibió hablar en los púlpitos de las Iglesias Presbiterianas del Brasil. Luego de una crisis de fe se transformó en apóstata del cristianismo y crítico de la religión organizada. Es autor de libros y artículos sobre temas religiosos, educacionales, existenciales y libros infantiles, y profesor de varias universidades entre ellas la UNICAMP, donde recibió el título de Profesor Emérito.

Richard Shaull fue presidente de la Federación Universal de Movimientos Estudiantiles Cristianos (FUMEC), entre 1968 y 1972. Miembro de la Iglesia Presbiteriana Unida de los Estados Unidos. Sus primeros años de ministerio transcurrieron como misionero en Colombia y Brasil, y en los últimos años fue profesor de Misión Cristiana en el Seminario Teológico de Princeton (Estados Unidos). Desempeñó un papel clave en la Conferencia de Iglesia y Sociedad en Ginebra en 1966. Fue profesor visitante en el Instituto Ecuménico en Bossey, entre 1962 y 1963. Fue una de las mentes teológicas más creativas que planteó la cuestión de la revolución desde una perspectiva teológica. Falleció a los 82 años.

Emilio Castro, pastor metodista y teólogo de Uruguay, fue director de la comisión del CMI sobre Misión Mundial y Evangelización, y luego Secretario General del CMI entre 1985 y 1992. Fue parte del grupo de líderes protestantes en América Latina con puntos de vista progresistas sobre el cristianismo y el orden social (ver web del CMI).

Madathilparampil Mammen Thomas (1916-1996) fue un teólogo cristiano indio, militante y pensador social. En 1978 la Universidad de Uppsala le otorgó el título de Doctor Honoris Causa. Fue criado en una familia perteneciente a la Iglesia Ortodoxa India ${ }^{3}$. Fue profesor en una escuela de esa iglesia, donde los profesores utilizan para pagar parte de su magro salario a los estudiantes de familias pobres. En 1937, comenzó

${ }^{3}$ Esta iglesia también es conocida como Iglesia Ortodoxa de Malankara, Iglesia Ortodoxa del Oriente, Iglesia Ortodoxa Siria de Malankara, Iglesia Ortodoxa Siria del Oriente o Iglesia Mar Thoma. Es una iglesia cristiana fundada por Santo Tomás, quien llegó a la India en el año 52 dC y sufrió el martirio en el año 72 en la actual ciudad de Chennai, al sur de la India. 
un orfanato en Trivandrum (capital de Kerala). Allí tomó contacto con el movimiento independentista indio y el pensamiento marxista. Entre 1945 y 1947 fue secretario de la organización del Yuvajana Sakhyam (el ala juvenil de la Iglesia Ortodoxa India). Sin embargo, su opción por el cristianismo y el marxismo causó que su iglesia no le permitiera ordenarse pastor, en tanto que el partido comunista lo expulsó por su fe religiosa. De todos modos se recibió de teólogo. Publicó varios libros sobre religión y participó del movimiento ecuménicó. Primero entre 1946 y 1951 se desempeñó en FUMEC y luego entre 1968 y 1975 fue presidente del Comité Central del Consejo Mundial de Iglesias (CMI). Con respecto a su actividad política, entre 1991 y 1993 fue Gobernador de Nagaland, un estado indio con un gran número de cristianos.

John Karefa-Smart (nació en 1915) es un político y líder del United National Peoples Party (UNPP) de Sierra Leona. En 1936, se licenció en letras en la Universidad de Fourah Bay en Freetown (Capital de Sierra Leona). En 1940, se recibió de licenciado en ciencias en el Otterbein College en Ohio (EE.UU.). En 1944 se recibió de doctor con especialidad en cirugía en la Universidad McGill en Montreal (Canadá) y al año siguiente obtuvo un diplomado en Medicina Tropical en la misma universidad. Por último, en 1948, obtuvo un doctorado en salud pública en la Universidad de Harvard, en Boston, EE.UU. Fue profesor en universidades de Sierra Leona, Nigeria y Estados Unidos. Desde 1957 a 1964, Karefa-Smart fue miembro electo del Parlamento por el Distrito Tonkolili (Sierra Leona). También se desempeñó como Ministro de Tierras, Minas y Trabajo, Defensa, y como Ministro de Relaciones Exteriores. Desde 1965 hasta 1970, fue Subdirector General de la Organización Mundial de la Salud. En 1996, regresó a Sierra Leona como miembro del parlamento y candidato presidencial, pero perdió las elecciones presidenciales de 1996 y 2002.

Richard Andriamanjato (nació en 1930) es un expolítico malgache. Entre 1950 y 1957 estudió en Francia. Después de dejar la educación, Andriamanjato se convirtió en un pastor. Comprometido con el movimiento independentista de Madagascar. En 1957, asistió a la Conferencia de Bandung y se convirtió en la figura principal del movimiento independentista opuesto a Filiberto Tsiranana. Andriamanjato participó en el Consejo de la Federación Protestante de Madagascar (FJKM), fue Presidente del Consejo de las Iglesias de África, fue miembro del Consejo Mundial de Iglesias de Ginebra y director del Instituto Cristiano para la Paz. Paralelamente, era un convencido comunista. En 1958, fundó el Parti du Congrès de l'indépendence de Madagascar (Partido del Congreso de la Independencia de Madagascar), AKFM por su sigla en malgache ${ }^{5}$, muy vinculado al Partido Comunista francés y actuó como la principal oposición durante más de una década. En 1959, fue elegido alcalde de Antananarivo (capital de Madagascar). En 1972, apoyó el golpe militar del líder socialista Didier Ratsiraka ${ }^{6}$

\footnotetext{
${ }^{4}$ Algunos de sus libros fueron: The Nagas Towards A.D. 2000 and other Selected Addresses and Writings, Madras: Centre for Research on New International Economic Order, 1992; My Ecumenical Journey 1947-75, Trivandrum: Ecumenical Publishing Centre Private Limited, 1990; Adhunika Bharathathile Rashtreeya Prathyaya Sasthrangal (Traducción: La ideología política de la India moderna), Tiruvalla: Theological Literature Committee, 1983; Kraisthava Samuhya Dharmam: Oru Vedapusthaka Padanam (Traducción: Ética social cristiana: Una exploración Bíblica) Tiruvalla: Theological Literature Committee, 1972; The acknowledged Christ of the Indian Renaissance, London: SCM Press, 1969 (http:/ / drmmthomas.blogspot.com/search/ label/bibliography).

${ }^{5}$ Antoko'ny Kongresi'ny Fahaleovantenan'i Madagasikara.

${ }^{6}$ Ratsiraka fue Presidente de Madagascar entre 1975 y 1993, y de nuevo (pero a través de elecciones) entre 1997 y 2002.
} 
y en 1976 dirigió el AKFM en el Frente Nacional para la Defensa de la Revolución, la coalición de gobierno. Entre 1991 y 1993, fue co-presidente de la transición Comité de Recuperación Económica y Social, junto con Manandafy Rakotonirina. En 1993 se convirtió en el Presidente de la Asamblea Nacional de Madagascar, sirviendo en esa posición hasta 1998. Fue candidato a las elecciones presidenciales de 1996 pero perdió. Posteriormente dimitió de la vida pública.

Hiber Conteris (nació en 1933) escritor uruguayo y uno de los miembros fundadores de ISAL. Entre 1953 y 1959 estudió filosofía y letras en Buenos Aires (Facultad de Filosofía y Letras, UBA) y en Montevideo (Facultad de Humanidades y Ciencias, UDELAR). También estudió teatro en la Escuela Municipal de Arte Dramático de Montevideo en los años 1958 y 1959. Entre 1966 y 1968 realizó su doctorado de tercer ciclo en la Sorbonne, París, sobre Sociología de la Literatura. También realizó estudios de posgrado sobre ideología y ciencia en la Universidad de Lovaina, Bélgica, en 1973. Autor de numerosas novelas: Enterrar a los muertos (1959); Este otro lado del telón (1960); El desvio (1963); El socavón (1963); El asesinato de Malcolm X (1969); El Intruso (1986); Holiday Inn (1987); ¿Qué desea cenar? (1996); El cielo puede esperar (1998); Rastros de ceniza; (2001); Onetti en el espejo (2005); El Intruso (2008); entre otras.

La característica en común de estos puntos de corte es su militancia cristiana, además que tenían alrededor de treinta años cuando publicaron con Mauricio López y su gran expansión sobre el globo terrestre (a primera vista encontramos autores de África, Asia y América). Una de las causas de lo último puede ser que todos los autores habían viajado a Europa y en algunos casos también a América, África o Asia. Otra característica es que todos son hombres, en realidad toda la red está conformada casi exclusivamente por hombres. Este hecho está en sintonía con el resto de las redes de intelectuales de occidente de mediados del siglo XX, pero es más común en las redes de intelectuales teológicas cristianas en las que el rol de las mujeres es marginal aún hoy. La principal diferencia radica en la importancia política que adquirieron en sus países de origen, la cual varió entre muy alta para los autores de África y Asia; a moderada y leve para los americanos. La diferencia de importancia se debe al apoyo a los procesos de descolonización de Asia y África, por parte de los militantes ecuménicos del Consejo Mundial de Iglesias. Los puntos de corte repiten las principales características de Mauricio López: varones adultos jóvenes que realizaron viajes intercontinentales como parte de su desarrollo intelectual y militancia cristiana ecuménica.

\section{CONCLUSIONES}

A partir de los títulos de las publicaciones puede afirmarse que la red de coautorías encontrada es de claro corte político-religioso. Mauricio López escribió principalmente sobre aspectos de la militancia político-religiosa tales como reflexiones teológicas o filosóficas, diagnósticos del movimiento estudiantil en América Latina, relatos de encuentros con organizaciones cristianas y reseñas de libros. Un aspecto llamativo 
de las publicaciones en que Mauricio López fue coautor es su bajo impacto local en contraposición a su alta divulgación global. López publicó mayormente al exterior de la Argentina (el 69\% de sus trabajos los escribió en inglés o en alemán), por esta razón sus escritos no son muy conocidos en su patria. En el país rioplatense es más bien recordado por ser el primer rector de la Universidad Nacional de San Luis, por su apoyo a los exiliados chilenos y por el impacto que su desaparición tuvo en la opinión pública internacional.

La red de coautorías de López no puede entenderse sin sus viajes. Durante su vida visitó a organizaciones de 25 países americanos, 8 naciones europeas y la India. Su red fue tejiéndose a través de contactos personales y sus publicaciones en coautoría emergieron de estas instancias de discusión. Como resultado escribió junto a europeos (que son el 42,4\% de la red), americanos (el 41,3\%), africanos y asiáticos (el 16,3\% entre ambos) conformando una red de 92 intelectuales. Gran parte de los recorridos por el globo fueron financiados por el Consejo Mundial de Iglesias, primero cuando López fue secretario para América Latina de FUMEC y luego como secretario adjunto de Iglesia y Sociedad. En este periodo el Consejo Mundial de Iglesias, además de vincular a los pensadores latinoamericanos con Europa (como había sucedido históricamente con las religiones cristianas), creó lazos entre autores de países periféricos de América, África y Asia, rompiendo el monopolio del nexo entre intelectuales del norte y del sur (en otras palabras entre el centro y la periferia). En este sentido, la red de coautorías de Mauricio López ha presentado la particularidad de tener vínculos con importantes actores de la vida política de África y la India. Tales son los casos John Karefa-Smart de Sierra Leona (fue ministro y candidato a presidente en dos oportunidades en su país y Subdirector General de la Organización Mundial de la Salud); Richard Andriamanjato de Madagascar (fue un importante militante del movimiento independentista de su país, alcalde de la capital de Madagascar y Presidente de la Asamblea Nacional) y M.M. Thomas de India (fue Presidente del CMI y Gobernador del Estado de Nagaland). Del mismo modo el Consejo Mundial de Iglesias fortaleció los vínculos entre el Bloque del Este y del Oeste en el contexto de la Guerra Fría lo que le permitió a Mauricio López vincularse con los países socialistas, principalmente a través de la Conferencia para la Paz Cristiana. Esto explica los escritos de López en alemán y el gran porcentaje de europeos en su red, algunos de ellos de gran renombre como el ortodoxo ruso Metropolitan Nikodim, famoso por su actuación en el acuerdo secreto con el Vaticano para facilitar la participación de su iglesia en el Concilio Vaticano Segundo a cambio de evitar la condena a los gobiernos socialistas ateos.

Respecto a sus miembros, la red aparece conformada por una mayoría de estudiantes universitarios y profesionales jóvenes, todos cristianos y varones. Esto ilustra el rol que ha ocupado la mujer en las religiones cristianas caracterizadas por una alta hegemonía del varón. Situación que ha variado según la organización religiosa. En la Iglesia Católica Apostólica Romana, por ejemplo, los altos cargos de decisión son monopolizados por hombres (obispos, cardenales y hasta el Papa), otras instituciones como las Iglesias Evangélicas Metodistas han permitido el ordenamiento de Pastoras. A pesar de estos matices, la mujer casi no ha ocupado roles de liderazgos dentro del cristianismo, ni en la práctica ni en las reflexiones teológicas de la época (a pesar de 
algunas excepciones como la teología feminista de la liberación).

En cuanto a los cliques encontrados en la red, el trabajo de investigación realizado permite afirmar que son en realidad la cristalización de espacios de militancia y que actuaron como colegios invisibles. La perspectiva holística de la metodología del ARS descentra al actor investigado (en este caso Mauricio López) y lo sitúa en su contexto vinculándolo con actores sincrónicos y formulando nuevas preguntas. La conformación de "colegios invisibles" es una de ellas. Estos son grupos de intelectuales que trabajan sobre temas similares e intercambian información por diferentes medios. La perspectiva sobre los colegios invisibles puede variar según lo analicemos desde un autor, desde todos los autores que conforman el colegio, desde la evolución temporal y/o espacial del colegio o desde los procesos de liderazgo al interior del mismo (Peñaranda y Quiñones, 2004; Figeredo, 2004). Desde los estudios bibliométricos se los define operativamente como al conjunto de autores que presentan al menos una publicación conjunta. En este trabajo, la red encontrada estuvo compuesta por académicos que además eran militantes sociales. Esto se debe a que la red de coautoría en torno a Mauricio López emerge principalmente como una cristalización de la militancia político-religiosa de sus miembros. Por esa razón, al analizar los cliques que la integran, su conformación aparece ligada a espacios de militancia previa (de ahí los nombres que se les asignó a los mismos). En este contexto, los colegios invisibles no sólo incluyeron intercambios de reflexiones sociológicas, filosóficas y teológicas, sino también de experiencias de participación política (al interior de las iglesias a la que pertenecían sus miembros y también en los espacios políticos de sus países). De hecho, puede inferirse que los cliques encontrados: FUMEC, CMI, Conferencia de Ginebra, LMC, ISAL- CMI y Conferencia para la Paz Cristiana, actuaron como colegios invisibles para los miembros de la red.

Finalmente, nos detendremos en los puntos de corte entre los cliques. Su importancia radica en que vinculan a dos o más cliques y que para hacerlo, han publicado con más de un subgrupo de la red. Los puntos de corte tienen las mismas características que López: varones que realizaron viajes intercontinentales como parte de su desarrollo intelectual y su militancia cristiana ecuménica y que cuando publicaron tenían alrededor de treinta años (durante la descripción de los puntos de corte, nos preguntamos si esto no permitiría rescatar la utilidad del olvidado concepto de "generación" ahora en desuso pero que fue muy utilizado en la historia del arte). A excepción de Richard Shaull de los Estados Unidos, el resto nació en países periféricos ${ }^{8}$. Tomando como base la descripción de la biografía de los ocho puntos de corte podríamos afirmar que en su mayoría, los coautores latinoamericanos, africanos y el único caso asiático de la red tuvieron una trayectoria de militancia parecida a la de Mauricio López. Primero fueron miembros activos en sus iglesias e inclusive muchos de ellos fueron pastores o sacerdotes. Luego tuvieron cargos de distinto rango en $\mathrm{La}$ Federación Universal de Movimientos Estudiantiles Cristianos (algunos fueron autoridades nacionales y otros continentales o participaron del comité central de Ginebra de

\footnotetext{
${ }^{8}$ Los puntos de corte en la red de coautoría son los latinoamericanos Julio de Santa Ana, Rubem Alves, Hiber Conteris y Emilio Castro; los africanos John Karefa-Smart y Richard Andriamanjato; el indio Madathilparampil Mammen Thomas y el estadounidense, Richard Shaull.
} 
FUMEC). Paralelamente, realizaron estudios de grado en sus países de origen y muchos de ellos, estudios de posgrado en Europa o Norteamérica. En una tercera etapa se unieron al Consejo Mundial de Iglesias como una instancia de militancia ecuménica superior (en realidad, la mayoría ya había comenzado a vincularse con el CMI a partir de FUMEC). Finalmente, luego de este periodo volvieron a sus países: los africanos (y también el autor indio) se integraron a la lucha por la descolonización de sus pueblos y posteriormente formaron parte de la clase política de sus países. Los latinoamericanos también fueron militantes políticos, algunos de ellos (no todos) se volcaron a la acción armada, como pasó con algunos miembros de ISAL, pero casi todos sufrieron la persecución de algún modo (la cárcel, el exilio o la desaparición). Esto último hizo que algunos regresaran a Europa. Como síntesis, se podrían plantear cuatro etapas sucesivas: 1) Alto compromiso al interior de sus iglesias, 2) Militancia en FUMEC, 3) Militancia en el CMI y 4) Participación en el escenario político de su país de origen (la segunda y tercera fase se realizó en paralelo a estudios de grado y de posgrados en el extranjero). Las trayectorias de militancias político-religiosa encontradas en europeos y norteamericanos son diferentes. Los primeros se encontraban inmersos en la reconstrucción de sus países y sus iglesias, luego de la Segunda Guerra Mundial, pero a los países de Europa del Este se les sumó la represión estatal a sus prácticas religiosas. En cuanto a los norteamericanos miembros de la red eran pastores que estuvieron realizando misiones en Latinoamérica.

Este trabajo ha ilustrado la utilidad de la metodología del análisis de redes sociales (ARS) empleada para el análisis de redes entre intelectuales. En este sentido, la reconstrucción de redes de coautorías (partiendo en este caso de un actor pero que podría hacerse a partir de una revista o una editorial) utilizando los softwares específicos ha demostrado su potencial capacidad para describir aspectos olvidados o retaceados por otras metodologías que parten solamente de las atribuciones individuales de los actores analizados.

\section{BIBLIOGRAFÍA}

Alcaide González, G.; Alonso Arroyo, A.; González de Dios, J.; Sempere, A.; Valderrama Zurián, J. y Benavent R. (2008), “Redes de coautoría y colaboración institucional en Revista de Neurología" Revista de neurología 46, 11 (Barcelona), pp. 642-651.

Alcaide González, Gregorio; Agulló Calatayud, Víctor; Valderrama Zurián, Juan Carlos y Benavent, Rafael Aleixandre (2009), “Participación de la mujer y redes de coautoría en las revistas españolas de Sociología", REIS. Revista Española de Investigaciones Sociológicas 126 (Madrid), pp. 153-166.

Chiroque-Solano, Richard y Padilla-Santoyo, Paola (2009), “Análisis de coautoría en la revista Biblios: Una aproximación desde Google Scholar". Biblios, pp. 33- 34.

De Carvalho Dias, Agemir. O movimento ecumênico no Brasil (1954-1994). Ao serviço da igreja e dos movimentos populares, (2007) Universidad Federal do Paraná: Tesis Doctoral en História. Biblioteca Digital Univ. Federal do Paraná www.agemir. 
com.br/O_movimento_ecum\%C3\%AAnico_no_Brasil_\%281954-1994\%29.pdf

Figueredo, E. (2004), "Los colegios invisibles" en Revista de la Sociedad Española del Dolor, 11, 6 (La Coruña), pp. 333-334.

Herrero, Reyes, (2000), “Glosario. Revisión crítica de la terminología propia del ‘social network analysis' y su traducción”. en Política y sociedad 33 (Madrid), pp. 199-206.

Iñiguez, Lupicinio; Juan Muñoz, Peñaranda, María Carmen y Martínez, Luz María (2006), “La psicología social en España: estructuras de comunidades" REDES 10, (Barcelona) artículo 3 (http:/ / revista-redes.rediris.es/).

Molina, José Luis; Muñoz Justicia, Juan y Domenech, Miquel (2002), "Redes de publicaciones científicas. Un análisis de la estructura de coautorías" Revista Redes, 1. (Barcelona) artículo 3 (http:/ / revista-redes.rediris.es/).

Paredes, Alejandro, Mauricio Amilcar López. Biografía y escritos sobre las revoluciones en América Latina (2008) Mendoza: Qellqasqa. pp. 978-987.

Peñaranda Ortega, María y Quiñones Vidal, Elena (2004), “Una nueva representación gráfica de 'colegios invisibles': los Colegios Invisibles Geográficos. El caso de Shelley Chaiken y el Journal of Personality and Social Psychology" en Revista de historia de la psicología, 25, 4 (Valencia), pp. 197-210.

Potter, Philip y Wieser, Thomas Seeking and Serving the Truth. The First Years of the World Student Christian Federation (1997) WCC publications, Ginebra.

\section{Fuentes primarias}

Publicaciones de Mauricio López rastreadas en el Consejo Mundial de Iglesias (Ginebra- Suiza), Instituto Iberoamericano de Berlín (Alemania) e ISEDET (Buenos Aires -Argentina).

\section{Entrevistas}

de Santa Ana, Julio. Octubre de 2007, Ginebra, Suiza.

\section{Información on line:}

ALC (2006) (Agencia Latinoamericana y caribeña de comunicación) Konrad Raiser destaca aporte latinoamericano en conferencia sobre Iglesia y Sociedad. http: / / elistas.alcnoticias.org/lista/espanol_alc/archivo/indice/1781/msg/1808/ [leído el 3/8/09].

www.fumec-alc.org 
Redes ecuménicas a mediados del siglo XX.

Secretarios generales, consejo mundial de iglesias. http:/ / www.oikoumene.org/es/ quienes-somos/informacion-de-fondo/historia/secretarios-generales.html.

http:/ / drmmthomas.blogspot.com/search/label/bibliography

http:/ / www.dramaturgiauruguaya.gub.uy/obras/autores/hiber-conteris / 